\title{
Barriers to treatment of failed or interferon ineligible patients in the era of DAA: single center study
}

\author{
Kwang Il Seo', Byung Chul Yun', Weiquan James Li ${ }^{1,2}$, Sang Uk Lee', Byung Hoon Han', and Eun Taek Park \\ 'Department of Internal Medicine, Kosin University College of Medicine, Busan, Korea, ${ }^{2}$ Department of Gastroenterology and Hepatology, \\ Changi General Hospital, Singapore
}

Background/Aims: Interferon-based treatment is not appropriate for a large number of patients with chronic hepatitis $\mathrm{C}$ for various medical and social reasons. Newly developed directly acting antivirals (DAAs) have been used to treat chronic hepatitis C without severe adverse effects and have achieved a sustained viral response (SVR) rate of $80-90 \%$ with short treatment duration. We were interested to determine whether all patients who failed to respond to or were ineligible for interferon-based therapy could be treated with DAAs.

Methods: Medical records of patients with positive serum anti-hepatitis C virus (HCV) or HCV RNA between January 2009 and December 2013 were reviewed. Demographic, clinical, and treatment data were collected for analysis.

Results: A total of 876 patients were positive for both anti-HCV and HCV RNA. Of these, 244 patients were eligible for interferon, although this was associated with relapse in 39 (16\%) of patients. In total, 130 patients stopped interferon therapy (67\% adverse effects, $28 \%$ non-adherent, $4 \%$ malignancy, $1 \%$ alcohol abuse) and 502 patients were ineligible ( $66 \%$ medical contraindications, $25 \%$ non-adherent, $5 \%$ socioeconomic problems). Among 671 patients who were ineligible for or failed to respond to interferon therapy, more than $186(27.7 \%)$ could not be treated with DAA due to financial, social, or cancer-related conditions.

Conclusions: Newly developed DAAs are a promising treatment for patients with chronic hepatitis $C$ who are ineligible for or failed to respond to interferon-based therapy. Nevertheless, not all chronic hepatitis $\mathrm{C}$ patients can be treated with DAAs due to various reasons. (Clin Mol Hepatol 2017;23:74-79)

Keywords: Hepatitis C; Direct acting antivirals; Interferons

\section{INTRODUCTION}

Hepatitis C virus (HCV) is a major cause of chronic liver disease. Interferon therapy for chronic hepatitis $\mathrm{C}$ has a relatively low rate of sustained viral response (SVR) of only $10-20 \%$ after 48 weeks.' Pegylated interferon (PegIFN) combined with ribavirin was the main therapy for chronic hepatitis C with an overall SVR rate of about $40-60 \%$ for genotype 1 and $70-80 \%$ for genotypes 2 and $3 .{ }^{2,3}$ However, the combination of standard PegIFN- $\alpha$ and ribavirin has strict indications and various adverse effects. In clinical situations, only $20-30 \%$ of patients with chronic hepatitis $C$ are treated with PegIFN- $\alpha$ and ribavirin. ${ }^{4}$ Moreover, less than $5 \%$ of the HCV-infected population worldwide is aware of their infection, and only about $3 \%$ are treated with antiviral therapy.

\section{Abbreviations:}

DAAs, direct acting antivirals; $H C V$, hepatitis C virus; RNA, ribonucleic acid

\section{Corresponding author : Byung Chul Yun}

Department of Internal Medicine, Kosin University College of Medicine, 262 Gamcheon-ro, Seo-gu, Busan 49267, Korea

Tel: +82-51-990-5060, Fax: +82-51-990-5055

E-mail:ybchepa@ns.kosinmed.or.kr 
Recently developed directly acting antivirals (DAAs) therapies have shown remarkable SVR rates above $90 \%$ with minimal adverse events. HCV eradication is suggested as a realistic goal of treatment by many hepatologists, ${ }^{6}$ but it remains a substantial challenge ${ }^{5}$ because acute and chronic hepatitis C virus infection are largely asymptomatic and comprehensive screening programs are rare in highly endemic regions. Controlling HCV requires a combination of identifying infections, proper treatment, and effective prevention. Despite highly efficient oral DAA therapy, the clinical eradication of HCV has been suggested to be difficult due to various unexpected barriers to treatment. Therefore, the aim of our study was to investigate the proportion of patients with and reasons for PegIFN- $\alpha$ and ribavirin ineligibility or failure, and to determine if these factors would also potentially become a barrier to treatment with DAAs.

\section{MATERIALS AND METHODS}

\section{Study population}

The medical records of 1,277 patients who were tested for antiHCV antibodies or HCV ribonucleic acid (RNA) levels at Kosin University Gospel Hospital in Busan, Korea from January 2009 to December 2013 were reviewed. All patients were evaluated by one of three hepatologists for diagnosis and treatment planning. Decisions about treatment were made according to the Korean Asso- ciation for the Study of the Liver clinical practice guidelines.

The study exclusion criteria were decompensated cirrhosis, poorly controlled psychiatric disorder, extra-hepatic transplantation, autoimmune disease, uncontrolled thyroid disease, symptomatic cardiopulmonary disease, uncontrolled diabetes, uncontrolled anemia (hemoglobin $<10 \mathrm{~g} / \mathrm{dL}$ ), neutropenia (absolute neutrophil count $<750 / \mathrm{mm}^{3}$ ), thrombocytopenia (platelet $<50,000 / \mathrm{mm}^{3}$ ), active alcohol or drug use, cancer or unwillingness to undergo treatment.

\section{Study design}

The variables collected were treatment initiation, treatment results and reasons for stopping or not receiving treatment. Patients were classified as noncompliant if they had no follow-up visit or missed visits frequently not enough to receive proper treatment. Statistical analysis was conducted using SPSS software version 23.0 (SPSS Inc., Chicago, IL, USA) and $P$-value $<0.05$ was considered to be statistically significant.

\section{RESULTS}

\section{Baseline characteristics}

Between 2009 and 2013, anti-HCV antibody or HCV RNA labo-

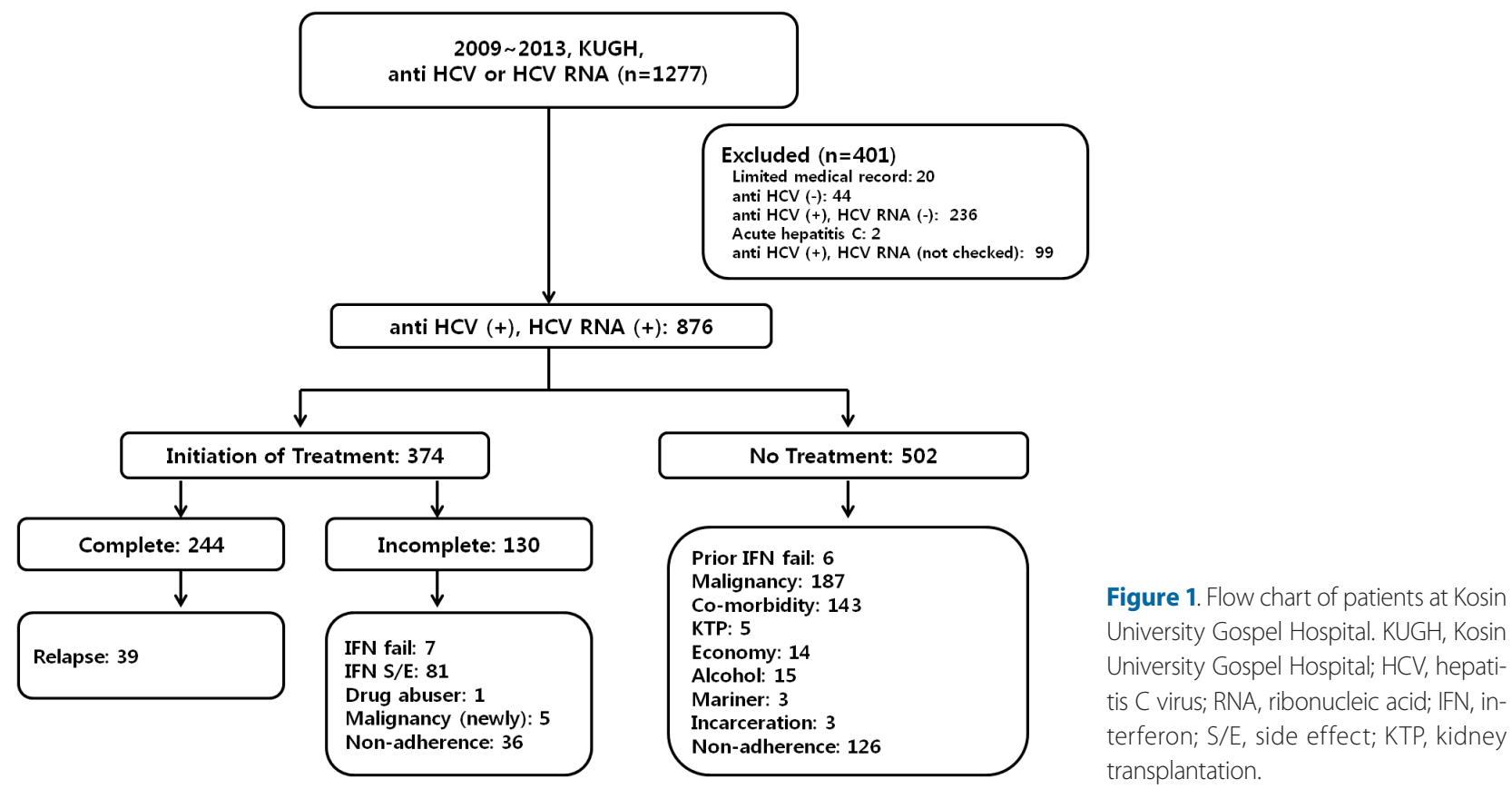


ratory tests were performed for 1,277 patients at Kosin University Gospel Hospital. Of these, 401 patients were excluded from this study. Two patients were diagnosed with acute hepatitis C, 44 were negative for anti-HCV antibody, 236 were positive for antiHCV antibody but negative for HCV RNA, and 20 had limited medical records (Fig. 1). Despite being positive for anti-HCV antibody, 99 patients did not have HCV RNA checked because of diagnosed malignancy $(n=57)$, severe medical or psychiatric illness

Table 1. Baseline characteristics of treated and non-treated patients

\begin{tabular}{|c|c|c|c|}
\hline & $\begin{array}{l}\text { Non- } \\
\text { treatment } \\
(n=502)\end{array}$ & $\begin{array}{c}\text { Initiation of } \\
\text { treatment } \\
(n=374,42.6 \%)\end{array}$ & $P$-value \\
\hline Mean age (years) & $61.9(19-87)$ & $55.5(25-83)$ & $<0.001$ \\
\hline \multicolumn{4}{|l|}{ Sex, n (\%) } \\
\hline Male & $271(53.7)$ & $177(47.3)$ & \\
\hline Female & 231 (46.3) & 197 (52.7) & \\
\hline Genotype, n (\%) & & & 0.009 \\
\hline Type 1 (total) & 191 & 183 & \\
\hline 1 & 9 & 8 & \\
\hline $1 a$ & 5 & 2 & \\
\hline $1 b$ & 177 & 173 & \\
\hline Type 2 (total) & 164 & 185 & \\
\hline 2 & 30 & 37 & \\
\hline $2 \mathrm{a} / 2 \mathrm{c}$ & 125 & 125 & \\
\hline $2 b$ & 9 & 23 & \\
\hline $1 \& 2$ & 5 & 0 & \\
\hline Other type & 2 & 6 & \\
\hline NA & 140 & 0 & \\
\hline
\end{tabular}

NA, not available. $(n=21)$, financial status $(n=1)$, ongoing alcohol abuse $(n=2)$, and not following medical advice $(n=18)$.

A total of 876 patients were confirmed positive for both antiHCV antibody and HCV RNA and diagnosed with chronic hepatitis C. Three-hundred seventy-four patients (42.6\%) started interferon-based antiviral therapy. The patients who were treated with interferon therapy were younger than the non-treated group $(P<0.001)$. Genotype 2 was more prevalent in the treated group $(P=0.009)$ (Table 1). Two-hundred forty-four (28\%) patients completed treatment with combined standard PegIFN- $\alpha$ and ribavirin, and 39 (16\%) patients were diagnosed with HCV relapse. Overall treatment response irrespective of genotype was $72.9 \%$. Virological responses according to genotype were similar to previous reports. ${ }^{3}$ SVR for genotype 1 was $66.9 \%$, genotype 2 was $77.8 \%$ (Table 2).

\section{Reasons for stopping or preventing interferon-based therapy}

One hundred thirty patients did not complete 3 or 6 months of treatment for various reasons: adverse effects of interferon-based therapy $(n=81,62 \%)$, poor response to interferon-based therapy $(n=7,5 \%)$, noncompliance with medical advice or loss to followup ( $n=36,27 \%)$, newly developed malignancy $(n=5,4 \%)$, or active alcohol abuse ( $n=1,0.7 \%$ ) (Fig. 2). A total of 502 patients could not initiate interferon-based antiviral therapy because of malignancy ( $n=187,37 \%)$, medical contraindications $(n=143$, $28 \%$ ), not following medical recommendations ( $n=126,25 \%)$, financial status ( $n=14,3 \%)$, ongoing alcohol use $(n=15,3 \%)$, failing prior interferon-based therapy $(n=6,1 \%)$, kidney transplanta-

Table 2. Virologic responses according to genotype

\begin{tabular}{|c|c|c|c|c|}
\hline & Complete Tx. $(n=244)$ & $\operatorname{ETR}(n=205)$ & $\begin{array}{c}\text { SVR }(n=178, \\
178 / 244=72.9 \%)\end{array}$ & Relapse $(n=39)$ \\
\hline \multicolumn{5}{|l|}{ Genotype, n (\%) } \\
\hline Type 1 (total) & 99 & 77 & $66(66 / 99=66.9 \%)$ & 22 \\
\hline 1 & & 3 & 2 & 2 \\
\hline $1 \mathrm{a}$ & & 1 & 1 & 1 \\
\hline $1 \mathrm{~b}$ & & 73 & 63 & 19 \\
\hline Type 2 (total) & 140 & 123 & 109 (109/140=77.8\%) & 17 \\
\hline 2 & & 26 & 23 & 9 \\
\hline $2 \mathrm{a} / 2 \mathrm{c}$ & & 82 & 72 & 6 \\
\hline $2 b$ & & 15 & 14 & 2 \\
\hline Other type & 5 & 5 & 3 & \\
\hline
\end{tabular}

ETR, end-of-treatment response; SVR, sustained virological response. 
Reasons for stopping treatment $(n=130)$

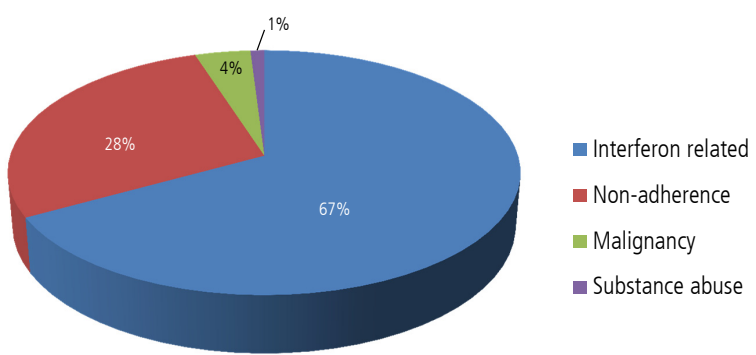

Figure 2. Reasons for stopping interferon-based therapy.

Reasons for not treating patients $(n=502)$

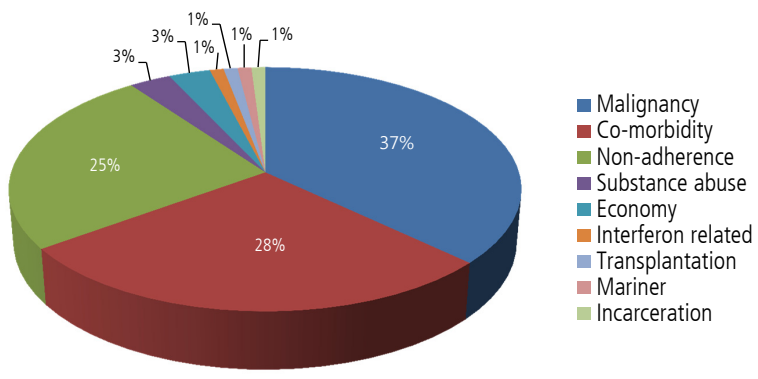

Figure 3. Reasons for not administering interferon-based therapy.

tion $(n=5,1 \%)$, mariner ( $n=3)$, and incarceration ( $n=3)$ (Fig. 3).

Malignancy was the most common reason for stopping or not receiving interferon-based therapy for chronic hepatitis $C$ in our study. Of the 192 patients with malignancy, 156 (81\%) had hepatocellular carcinoma (HCC), 15 (8\%) had gastrointestinal cancer, and $6(3 \%)$ had pancreaticobiliary cancer (Table 3). Of the 148 patients who did not receive interferon-based antiviral therapy due to medical conditions, 49\% had advanced liver cirrhosis, 10\% were elderly, $10 \%$ had a poorly controlled psychiatric disorder, $8 \%$ had symptomatic cardiac disease, $5 \%$ had end-stage renal disease, $3 \%$ had kidney transplantation, 3\% had uncontrolled diabetes, 3\% had hematologic disease, and 3\% had severe infection (Fig. 4).

Of the 192 patients who did not initiate or complete interferonbased antiviral therapy for nonmedical reasons, 162 (26\%) were noncompliant with evaluation, education, and visit appointments; 16 (3\%) were actively abusing alcohol, and $14(2 \%)$ had financial difficulties. Other complicating reasons included mariner or incarceration (Fig. 5).

\section{DISCUSSION}

The prevalence of blood-borne HCV infection is estimated to be
Table 3. Proportion of malignancies that resulted in no treatment or stopped treatment

\begin{tabular}{ll}
\hline Malignancy & $\mathbf{n = 1 9 2}$ \\
\hline Hepatocellular carcinoma & $156(81)$ \\
\hline Gastrointestinal & $15(8)$ \\
\hline Stomach cancer & 7 \\
\hline Colon cancer & 6 \\
\hline Esophageal cancer & 2 \\
\hline Pancreaticobiliary cancer & $6(3)$ \\
\hline Female malignancy & $7(4)$ \\
\hline Breast cancer & 4 \\
\hline Gynecologic cancer & 3 \\
\hline Etc. & $8(4)$ \\
\hline Lung cancer & 2 \\
\hline Thyroid cancer & 2 \\
\hline Renal cancer & 2 \\
\hline NHL & 1 \\
\hline Skin malignancy & 1 \\
\hline
\end{tabular}

Values are presented as $n(\%)$.

NHL, non-hodgkin's lymphoma.

\section{Medical conditions preventing treatment $(n=148)$}



- Advanced cirrhosis
Transplantation
ESRD
- Heart
Uncontrolled DM
Neurology
Psychiatric disorder
Allergy
Hematology
Infection
Old age

Figure 4. Combined medical conditions preventing treatment. DM, diabetes mellitus; ESRD, end stage renal disease.

about 3\% worldwide. Although DAAs that target nonstructural protein of HCV have potent antiviral efficacy and few adverse effects, the eradication of HCV is supposed to be difficult because most acute and chronic HCV infection is asymptomatic, and screening programs are limited in most countries. In a prospective, multicenter cohort at five university hospitals from January 2007 to December 2011, 1,173 patients age >18 years who were positive for anti-HCV antibody were enrolled to investigate the epidemiological and clinical characteristics of HCV infection of Korea. The rate of antiviral therapy for HCV was $42.8 \%$ of the HCV cohort. ${ }^{7}$ At our center, 876 patients were diagnosed in a chronic viremic state, and 374 (42.6\%) were treated with interfer- 
IFN ineligible or failed $(n=671)$

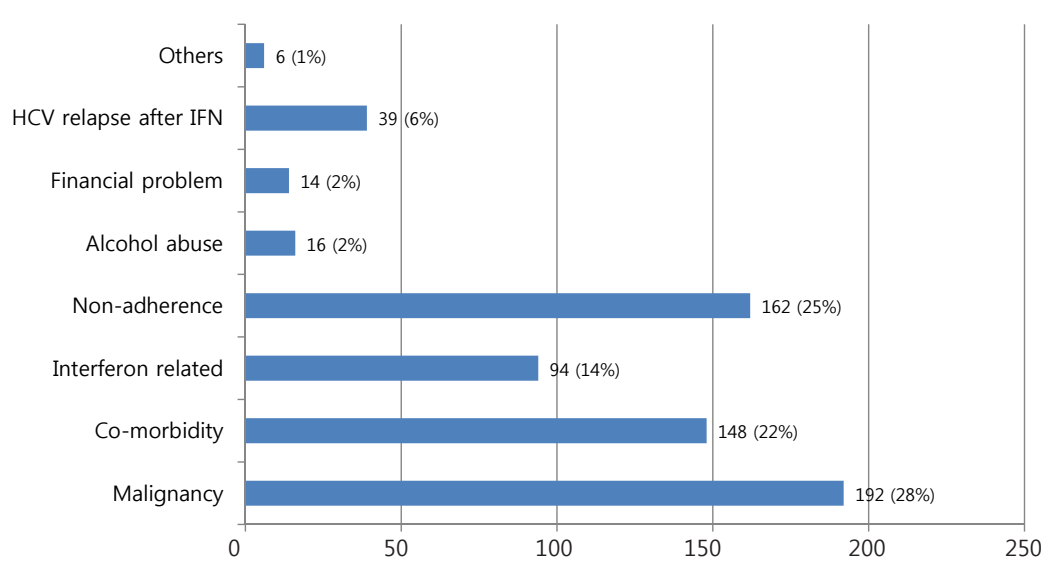

Figure 5. Patients who were ineligible for or failed to respond to interferon-based therapy. IFN, interferon. on-based therapy.

PegIFN- $\alpha$ and ribavirin combination therapy have absolute contraindications including uncontrolled depression; psychosis or epilepsy; pregnancy or couples unwilling to use adequate contraception; severe concurrent medical diseases; and co-morbidities including retinal disease, autoimmune thyroid disease, and decompensated liver disease. ${ }^{8}$ After considering the contraindications for interferon-based therapy, a large number of patients diagnosed with chronic hepatitis $C$ could not have treatment. Therefore, the proportion of patients initiating treatment was $31.0 \%$ in Swiss cohort study, ${ }^{9}$ and $33.0 \%$ in Danish cohort study. ${ }^{10}$ According to Falck-Ytter et al. ${ }^{4}, 72 \%$ of chronic hepatitis C patients were not treated. Of these, $37 \%$ did not adhere to medical recommendations, $34 \%$ were medically or psychologically ineligible, $13 \%$ had ongoing alcohol or drug abuse, and $11 \%$ refused treatment. Only $28 \%$ of chronic hepatitis C patients were treated and $13 \%$ had a sustained viral response. Narasimhan et al. ${ }^{11}$ retrospectively reviewed the charts of all HCV patients who underwent liver biopsies. About $60 \%$ were not treated with interferonbased therapy because of loss to follow-up or non-compliance $(31 \%)$, patient preference $(22 \%)$, etc. In other words, chronic hepatitis $\mathrm{C}$ treatment largely depended on patients' intention to treat, not medical decisions made by doctors. Restrepo et al. ${ }^{12}$ reported that approximately $85 \%$ of patients co-infected with HCV and human immunodeficiency virus were not treated. Of these, $40 \%$ were noncompliant with medical schedules, $15 \%$ were actively abusing drugs or alcohol, and $15 \%$ refused antiviral treatment.

In our study, 876 patients were diagnosed with chronic hepatitis C having positive results for both anti-HCV and HCV RNA. Of the 876 patients, 244 were eligible for interferon, but 39 (16\%) diagnosed with HCV relapse. 632 patients could not be treated appropriately with interferon-based antiviral therapy (Fig. 1). The reasons for stopping or not receiving interferon-based antiviral treatment include malignancy $(30 \%)$, co-morbidity $(23 \%)$, interferon-related reasons (15\%) and non-adherence (26\%).

The most prevalent malignancy in our cohort was HCC (81\%) (Table 2). In the interferon era, HCC was a relative contraindication for antiviral therapy; however, treating chronic hepatitis $C$ with interferon was considered to reduce HCC recurrence and improve survival. ${ }^{13}$ The recent introduction of DAA has been expected to reduce the incidence of HCC in HCV-related cirrhotic livers. Unfortunately, DAA-induced clearance of HCV was not able to reduce the occurrence of $\mathrm{HCC}$ in patients with $\mathrm{HCV}$-related cirrhotic livers. ${ }^{14}$ In spite of DAA treatment, high rate of early HCC recurrence was noted in patients previously treated for $\mathrm{HCC}^{14,15}$ Therefore DAA therapy in HCC patients has yet to be determined.

Patients with severe co-morbidities could not be treated with interferon-based therapy because of possible severe adverse effects. About $49 \%$ of patients had advanced or decompensated liver cirrhosis that was a contraindication to interferon therapy (Fig. 4). However, an oral DAA regimen could be safe and highly effective in treating patients who are ineligible for interferonbased antiviral therapy due to HCV-related cirrhosis, decompensation. In addition to advanced liver diseases, DAA could be administered safely in patients with symptomatic cardiovascular disease, chronic renal disease, uncontrolled diabetes, extra-hepatic transplantation and psychiatric disorders. ${ }^{16}$

Non-adherence, financial problems and alcohol abuse are also reasons to prevent antiviral therapy. With short treatment duration and all oral regimens, improved compliance is expected in patients using DAA. On the other hand, DAA is so expensive that it would be difficult for a physician to prescribe to all chronic hep- 
atitis C patients. Therefore, a part of compliance and socioeconomic problems would be remained in DAA era.

Therefore, we could suppose that at least $42 \%$ of patients who were ineligible or failed to interferon-based antiviral therapy due to co-morbidity (22\%), interferon intolerability (14\%) and HCV relapse (6\%) would be treated with DAAs. However, a large portion of patients who were non-adherent (25\%), had alcohol abuse $(2 \%)$, or had financial concerns (2\%) would not be able to get a chance to use the DAA (Fig. 5). Unlike hepatitis B virus-related HCC, patients with HCC occurred with HCV $(23 \%$, 156/671) have not been determined to be treated with DAA. This study has some limitations, including a retrospective design and that it was conducted in a single, tertiary hospital. The combined co-morbidity and malignancy rates were higher than in other Korean studies. ${ }^{7,17}$ Therefore, the results cannot be accepted generally. In spite of the limitation, this study has a clear message considering limitation of DAA therapy in chronic hepatitis C patients who were ineligible or failed to interferon based antiviral therapy.

In conclusion, only $27.7 \%$ of patients diagnosed with chronic hepatitis $C$ in our study were treated with an interferon-based regimen. With the advent of DAAs, at least $42 \%$ of patients who were ineligible or failed to interferon and experienced HCV relapse would be able to use DAA. However nonmedical reasons, including noncompliance, financial problems, substance abuse and hepatocelluar carcinoma remain obstacles to treat chronic hepatitis C. Our study suggests that, in spite of DAA development, eradicating HCV would be difficult due to various unexpected reasons.

\section{Conflicts of Interest}

The authors have no conflicts to disclose.

\section{REFERENCES}

1. Pawlotsky JM, Feld JJ, Zeuzem S, Hoofnagle JH. From non-A, non-B hepatitis to hepatitis C virus cure. J Hepatol 2015;62(1 Suppl):S87-S99.

2. Fried MW, Shiffman ML, Reddy KR, Smith C, Marinos G, Goncales $\mathrm{FL} J$ r, et al. Peginterferon alfa-2a plus ribavirin for chronic hepatitis C virus infection. N Engl J Med 2002;347:975-982.

3. Park SH, Park CK, Lee JW, Kim YS, Jeong SH, Kim YS, et al. Efficacy and tolerability of peginterferon alpha plus ribavirin in the routine daily treatment of chronic hepatitis $C$ patients in Korea: a multi- center, retrospective observational study. Gut Liver 2012;6:98-106.

4. Falck-Ytter Y, Kale H, Mullen KD, Sarbah SA, Sorescu L, McCullough AJ. Surprisingly small effect of antiviral treatment in patients with hepatitis C. Ann Intern Med 2002;136:288-292.

5. Cox AL. MEDICINE. Global control of hepatitis C virus. Science 2015;349:790-791.

6. Korean Association for the Study of the Liver. KASL clinical practice guidelines: management of hepatitis C. Clin Mol Hepatol 2016;22:76-139.

7. Seong MH, Kil H, Kim YS, Bae SH, Lee YJ, Lee HC, et al. Clinical and epidemiological features of hepatitis $C$ virus infection in South Korea: a prospective, multicenter cohort study. J Med Virol 2013;85:1724-1733.

8. European Association for Study of Liver. EASL recommendations on treatment of hepatitis C 2015. J Hepatol 2015;63:199-236.

9. Prasad L, Spicher VM, Zwahlen M, Rickenbach M, Helbling B, Negro F; Swiss Hepatitis C Cohort Study Group. Cohort profile: the Swiss Hepatitis C Cohort Study (SCCS). Int J Epidemiol 2007;36:731-737.

10. Hansen N, Obel N, Christensen PB, Krarup H, Laursen AL, Clausen $M R$, et al. Predictors of antiviral treatment initiation in hepatitis C virus-infected patients: a Danish cohort study. J Viral Hepat 2009;16:659-665.

11. Narasimhan G, Sargios TN, Kalakuntla R, Homel P, Clain DJ, Theise $N D$, et al. Treatment rates in patients with chronic hepatitis $C$ after liver biopsy. J Viral Hepat 2006;13:783-786.

12. Restrepo A, Johnson TC, Widjaja D, Yarmus L, Meyer K, Clain DJ, et al. The rate of treatment of chronic hepatitis $C$ in patients co-infected with HIV in an urban medical centre. J Viral Hepat 2005;12:86-90.

13. Singal AK, Freeman DH Jr, Anand BS. Meta-analysis: interferon improves outcomes following ablation or resection of hepatocellular carcinoma. Aliment Pharmacol Ther 2010;32:851-858.

14. Conti F, Buonfiglioli F, Scuteri A, Crespi C, Bolondi L, Caraceni P, et al. Early occurrence and recurrence of hepatocellular carcinoma in HCV-related cirrhosis treated with direct-acting antivirals. J Hepatol 2016:65:727-733.

15. Reig $M$, Mariño Z, Perelló $C$, Iñarrairaegui $M$, Ribeiro $A$, Lens $S$, et al. Unexpected high rate of early tumor recurrence in patients with HCV-related HCC undergoing interferon-free therapy. J Hepatol 2016;65:719-726.

16. Majumdar A, Kitson MT, Roberts SK. Systematic review: current concepts and challenges for the direct-acting antiviral era in hepatitis $C$ cirrhosis. Aliment Pharmacol Ther 2016;43:1276-1292.

17. Kim DY, Kim IH, Jeong SH, Cho YK, Lee JH, Jin YJ, et al. A nationwide seroepidemiology of hepatitis $C$ virus infection in South Korea. Liver Int 2013:33:586-594 